\title{
Commentary
}

\section{The state of psychosocial services in cancer care in the United States}

\author{
Teresa Deshields ${ }^{\prime *}$, Brad Zebrack² and Vicki Kennedy ${ }^{3}$ \\ 'Siteman Cancer Center at Barnes-Jewish Hospital and Washington University, St. Louis, MO, USA \\ ${ }^{2}$ University of Michigan School of Social Work, Ann Arbor, MI USA \\ ${ }^{3}$ The Cancer Support Community, WA, USA
}

* Correspondence to: Siteman Cancer Center, 4921 Parkview Place, MS: 90-35-703, St. Louis, MO 63/10, USA. E-mail: tld2593@bjc.org
Received: 31 January 2012

Accepted: 3 February 2012

\begin{abstract}
Objective: In 2009, the APOS commissioned a survey of its members and attendees of the annual meetings in 2008 and 2009 . The goal of the survey was to assess the scope of psychosocial support services for cancer patients in the USA.

Methods: Two hundred thirty-three individuals (27\% response rate) completed the survey, which included questions assessing the extent to which respondents' institutions provided informational and psychosocial support services and conducted screening for psychosocial distress.

Results: Respondents were primarily psychologists, although oncologists, nurses, social workers, and others were represented, as well. A broad array of informational and support services were endorsed as being provided to cancer patients, both at no charge or for a fee. Respondents identified social workers as the professionals most often providing psychosocial services to cancer patients. Respondents also indicated that most psychosocial services have not been tailored to fit a culturally diverse population. Furthermore, most of the organizations represented in the survey do not routinely screen cancer patients for psychosocial distress.

Conclusions: A broad range of psychosocial services are provided in cancer treatment settings; however, despite National Comprehensive Cancer Network and Institute of Medicine recommendations, routine screening for distress is not offered in a majority of cancer care organizations. Despite the racial, ethnic, cultural, and linguistic diversity of the US population, most organizations have not adapted their educational materials nor their psychosocial services to meet the needs of a diverse patient population.

Copyright @ 2012 John Wiley \& Sons, Ltd.
\end{abstract}

Keywords: psychosocial services; cancer; oncology; support services; diversity

\section{Introduction}

Psychosocial problems associated with cancer (e.g., depression, inadequate coping skills) can be effectively addressed by supportive care services and psychosocial interventions [1]. Nonetheless, the psychosocial needs of cancer survivors are not being met, according to a 2006 report by the Institute of Medicine (IOM) and National Research Council [2].

In 1995, Coluzzi and colleagues published results of a survey of supportive care services offered at National Cancer Institute (NCI)-designated cancer centers [3]. At that time, $68 \%$ of responding institutions offered counseling services and $90 \%$ offered support groups. Ninety percent had a spiritual care department. The source for most of the funding for these services was institutional funds.

The American Psychosocial Oncology Society (APOS) endeavored to update the previous survey and examine the current state of psychosocial services for cancer survivors. The primary goal of the new survey was to establish baseline evidence against which the field can measure progress on meeting the strategic imperatives promulgated in the IOM report, Cancer Care for the Whole Patient [1].

\section{Methods}

\section{Participants}

Data were collected from participants through an online survey (Survey Monkey) between November 2009 and February 2010. The link to the survey was e-mailed to APOS members and attendees at APOS annual conferences in the previous 2 years $(n=850)$.

\section{The survey}

The survey included questions assessing the extent to which respondents' institutions provided psychosocial services, including individual/family consultation, educational materials, support groups, and survivorship programs. The survey also solicited information about psychosocial screening procedures and barriers. The questions were drawn from the Coluzzi study [3], with additional questions added by the authors and APOS Board members. 


\section{Data analysis}

Given the primary focus of the survey to provide information about institutional practices (not individual behaviors), survey data from respondents from the same institution were condensed into a single institutional response. In cases where there were discrepancies, a set of decision rules was devised to determine the composite score. If one respondent from an institution reported their institution offered a program or service, or experienced a particular barrier to care, the composite case was coded as having that particular program, service, or barrier. In instances where respondents from the same institution reported differing numbers or proportions (e.g., of staff, services, barriers to care), the composite score reflected the greater number. With regard to costs, if one respondent reported services as being offered 'at no cost,' whereas another respondent from the same institution reported services as offered 'for a fee,' the composite response was coded as 'both at no cost and for a fee.'

Several open-ended questions were included in the survey. Open-ended responses were coded and then organized into categories determined by study authors.

\section{Results}

\section{Respondents}

The online survey was completed by 233 people (27\% response rate), representing 146 institutions across the USA. Thirty-six percent of respondents were psychologists, followed by social workers (19\%), and nurses
(13\%). Most respondents (79\%) provided clinical care and were APOS members $(61 \%)$.

Among the 146 institutions represented, 58 (40\%) were NCI-designated comprehensive cancer centers or large cancer treatment/research institutions. Forty (27\%) of the institutions were patient service or advocacy organizations. Thirty-three $(23 \%)$ of the institutions were community-based treatment centers (community hospitals, ambulatory care centers, small medical practices). Fifteen (10\%) of the institutions were nonmedical institutions not providing clinical care, such as university departments.

\section{Informational and psychosocial support services}

Psychosocial clinical care coverage: Across 131 institutions providing clinical services, the size of clinical staffs varied. Almost half of the institutions (41\%) reported 3-10 employees who provide psychosocial services. Approximately $19 \%$ of comprehensive centers, $39 \%$ of community-based treatment centers, and $28 \%$ of patient advocacy organizations reported staffs of fewer than three psychosocial professionals.

With regard to funding psychosocial care, most settings relied on multiple financial sources. Sixty-seven percent of comprehensive centers and $88 \%$ of community-based treatment sites are supported by general operating funds. Approximately, one-third of comprehensive centers (29\%), community-based centers (30\%), and patient service organizations (38\%) depend

Table I. Types of services $(n=|3|)$

\begin{tabular}{|c|c|c|c|c|c|c|}
\hline \multirow[b]{2}{*}{ Services } & \multicolumn{2}{|c|}{$\begin{array}{l}\text { Comprehensive cancer center } \\
\qquad n=58 \text { freq }(\%)\end{array}$} & \multicolumn{2}{|c|}{$\begin{array}{l}\text { Community-based treatment } \\
\text { centers } n=33 \text { freq }(\%)\end{array}$} & \multicolumn{2}{|c|}{$\begin{array}{l}\text { Patient service or advocacy } \\
\text { organizations } n=40 \text { freq (\%) }\end{array}$} \\
\hline & Offered & Charge $^{a}$ & Offered & Charge $^{a}$ & Offered & Charge $^{a}$ \\
\hline Professionally-led support groups & $44(76 \%)$ & $3(7 \%)$ & $26(79 \%)$ & $4(15 \%)$ & $20(50 \%)$ & $2(5 \%)$ \\
\hline Peer support network & $29(50 \%)$ & 0 & $21(64 \%)$ & 0 & $18(45 \%)$ & 0 \\
\hline Culturally-tailored support groups & $18(31 \%)$ & | (6\%) & $8(24 \%)$ & 0 & $13(33 \%)$ & | (8\%) \\
\hline Survivorship care program & $37(64 \%)$ & $5(14 \%)$ & $23(70 \%)$ & | (4\%) & $19(48 \%)$ & | (5\%) \\
\hline Individual counseling & $46(79 \%)$ & | | (24\%) & $25(76 \%)$ & $9(36 \%)$ & $22(55 \%)$ & $5(23 \%)$ \\
\hline Family counseling & $44(76 \%)$ & | ( $(25 \%)$ & $24(73 \%)$ & 7 (29\%) & $21(53 \%)$ & $4(19 \%)$ \\
\hline Psychiatric consultation & $44(76 \%)$ & $36(82 \%)$ & $20(61 \%)$ & $17(85 \%)$ & $4(10 \%)$ & $2(50 \%)$ \\
\hline Pastoral/ spiritual care & $43(74 \%)$ & 0 & $24(73 \%)$ & 0 & $7(18 \%)$ & 0 \\
\hline Genetic counseling & $43(74 \%)$ & $36(84 \%)$ & $23(70 \%)$ & $15(65 \%)$ & | (3\%) & 0 \\
\hline Fertility counseling & $38(66 \%)$ & 31 (82\%) & $12(36 \%)$ & $8(67 \%)$ & | (3\%) & 0 \\
\hline Employment counseling & $21(36 \%)$ & $4(19 \%)$ & II (33\%) & $2(18 \%)$ & $6(15 \%)$ & 0 \\
\hline Financial counseling & $43(74 \%)$ & $2(5 \%)$ & $26(79 \%)$ & 0 & $12(30 \%)$ & | (8\%) \\
\hline Exercise/ fitness program & $28(48 \%)$ & $7(25 \%)$ & $17(52 \%)$ & $5(29 \%)$ & $15(38 \%)$ & 0 \\
\hline Rehabilitation/ physical therapy & $41(71 \%)$ & 37 (90\%) & $23(40 \%)$ & $21(91 \%)$ & $3(8 \%)$ & 0 \\
\hline Diet/ nutrition program & $45(78 \%)$ & $21(47 \%)$ & $26(79 \%)$ & $8(31 \%)$ & $14(35 \%)$ & 0 \\
\hline Child care & $8(14 \%)$ & $2(25 \%)$ & $2(6 \%)$ & 0 & $2(5 \%)$ & 0 \\
\hline Transportation assistance & $35(60 \%)$ & $2(6 \%)$ & $21(64 \%)$ & | (5\%) & $7(18 \%)$ & 0 \\
\hline Educational materials & $46(79 \%)$ & $4(9 \%)$ & $28(85 \%)$ & $2(7 \%)$ & $25(63 \%)$ & | (4\%) \\
\hline Internet access & $38(66 \%)$ & 0 & $26(79 \%)$ & 0 & $19(48 \%)$ & 0 \\
\hline Patient education specialist & $31(53 \%)$ & $2(6 \%)$ & $22(67 \%)$ & 0 & $16(40 \%)$ & 0 \\
\hline Patient education resource center & $36(62 \%)$ & 0 & $28(85 \%)$ & 0 & $20(50 \%)$ & 0 \\
\hline Educational classes, workshops & $42(72 \%)$ & $5(\mid 2 \%)$ & $29(88 \%)$ & $5(17 \%)$ & $20(50 \%)$ & $2(10 \%)$ \\
\hline Informational website & $42(72 \%)$ & 0 & $28(85 \%)$ & 0 & $23(58 \%)$ & 0 \\
\hline Individual/family education consultation & $45(78 \%)$ & $26(58 \%)$ & $30(9 \mid \%)$ & $15(50 \%)$ & $27(68 \%)$ & $7(26 \%)$ \\
\hline
\end{tabular}

${ }^{a}$ Number/percentage that charge for the service of those institutions that offer the service. 
upon philanthropic funds. Forty-one percent of comprehensive centers reported charging fees for some services, compared with $30 \%$ of community treatment centers and $13 \%$ of patient service organizations. Between $17 \%$ and $26 \%$ of all institutions reported grants as a source of financial support.

Types of psychosocial services provided: Table 1 summarizes the psychosocial services offered. Services are provided both free of charge and feefor-service across different settings. A majority of community-based treatment centers and comprehensive cancer centers and about half of patient service organizations offer professionally-led support groups, with a subset charging a fee for this service. Also, psychiatric consultation, genetic counseling, fertility counseling, and rehabilitation therapy are most often offered by comprehensive centers and community treatment settings, with over $50 \%$ of those charging a fee.

Informational services (the provision of educational materials and community resources) were offered in most settings (see Table 1). Most institutions (63-85\%) provide educational materials, with very few charging for these materials. Most comprehensive centers and community sites and about half of patient service organizations provide free internet access for patients. Patient education resource centers were available at many comprehensive centers and community treatment sites and half of patient service organizations. Patient education specialists were more available at community sites than at comprehensive centers or patient service organizations. Most comprehensive centers and community sites and half of patient service organizations offered free educational classes or workshops.

A minority of responding organizations (31\% of comprehensive cancer centers, $24 \%$ of community treatment centers, and $33 \%$ of patient service organizations) offer culturally tailored support groups. Despite the widespread availability of informational and educational resources, over half of comprehensive centers $(52 \%)$, community centers $(64 \%)$, and patient service organizations $(58 \%)$ reported minimal adaptation of these materials $(<25 \%)$ to culturally or linguistically diverse patient groups. Only five comprehensive centers (9\%), four community centers $(12 \%)$, and two patient service organizations $(5 \%)$ indicated substantial adaptation of educational materials $(>50 \%)$.

Providers of psychosocial services: Although a variety of clinicians deliver psychosocial services (see Table 2), oncology social workers are most often responsible for delivery of services. In comprehensive cancer centers and community-based sites, social workers were most often identified as the providers of resource referral, case management, community outreach/education, and patient navigation. With regard to counseling services, social workers were most often identified across all sites as the providers of employment and vocational assistance, individual/family counseling, and telephone counseling. Psychologists were also identified as providers of individual/family counseling and telephone counseling.

Social workers were also most often identified as providing assistance with insurance, finances, housing, transportation, and legal resources, as well as providing psycho-educational programs. Psychologists provide complementary medicine services and also psycho-educational programs. In patient service organizations, social workers were most likely to provide all such services, as well as online information and referral.

Greater variety was observed in the delivery of lifestyle and health behavior interventions. In comprehensive cancer centers, nurses most often provided sexual health counseling, smoking cessation services, and nutrition information/counseling. Physicians and psychologists were also likely to provide sexual health counseling and smoking cessation services. Social workers were more likely to organize activities, such as survivorship events, or deliver appearance/body image programs. In community care sites, nurses were more likely to perform counseling related to sexual health, nutrition, and exercise/fitness, whereas social workers were again more likely to provide appearance/body image programs and organize patient activities. In patient service organizations, social workers and psychologists were most likely to be identified as providing sexual health counseling and appearance/body image programs and organizing patient activities, whereas nurses were endorsed as also providing sexual health counseling and appearance/body image programs but more likely to provide nutrition information/counseling.

The results indicate that most psychosocial services are not tailored for culturally diverse populations. Three fourths of institutions reported having adapted little $(<25 \%)$ of their psychosocial and lifestyle/behavioral health services in this manner. Only $11 \%$ of institutions had tailored many $(>50 \%)$ programs for multilingual and multicultural populations.

Barriers for psychosocial care for minority patients: Survey respondents listed three significant barriers to psychosocial care for patients from racial, ethnic, or linguistic minority groups: finances $(22 \%)$, language $(21 \%)$, and culture (20\%). Comprehensive cancer centers and community-based treatment centers endorsed finances (17\% and $36 \%$, respectively) and cultural issues (14\% and $30 \%$, respectively) as the most significant barriers to psychosocial care. Patient service organizations were more likely to endorse language (35\%) and geography/transportation (30\%) as significant barriers.

\section{Psychosocial screening}

Only $67(51 \%)$ of the organizations offering clinical care conduct routine psychosocial screening for new cancer patients. Twenty-five comprehensive centers (43\%), 22 community-based practices (67\%), and 19 


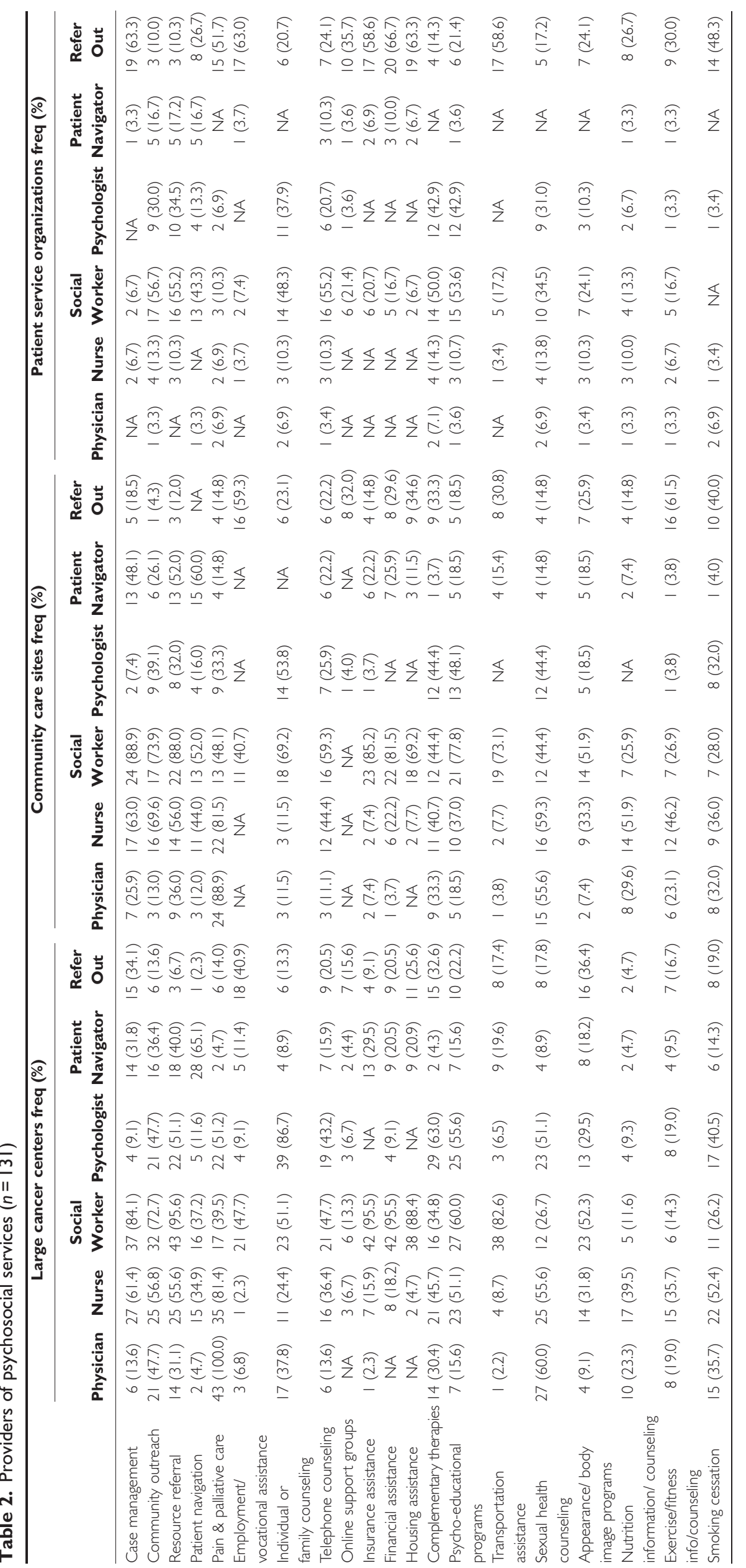


patient service organizations $(48 \%)$ conduct such screening. Open-ended interviews were the most common approach to psychosocial assessment $(88 \%$ of comprehensive centers, $82 \%$ of community-based centers, and $100 \%$ of patient service organizations), followed by administration of a distress measure (72\%, $68 \%$, and $42 \%$, respectively). Of the 64 institutions reported as not conducting psychosocial screening, the most common barrier was inadequate staffing, with 26 institutions (41\%) indicating this to be the case. Lack of administrative support (28\%) and lack of funding $(23 \%)$ were the next most common barriers. Twelve respondents (19\%) indicated that their institutions did not have a strategy to implement psychosocial screening and six cited staff resistance (9\%).

\section{Discussion}

Consistent with the findings of the 2008 IOM report [1], a broad range of supportive services are offered for cancer patients. Although a multidisciplinary cohort of oncology health professionals delivers these services, the diverse sample of survey respondents (nurses, social workers, psychologists, chaplains) agreed that social workers were the primary providers of oncology psychosocial services. Most respondents indicated that support services are offered at no charge, with funding from a variety of sources, most notably institutional funds. The ratio of funding sources varied by setting, with comprehensive cancer centers being more diversified but relying most heavily on institutional funds and fee for service. Holland has noted the inadequacy of reimbursement for billed psychosocial services [4], a difficulty for organizations that depend on fee for service income. The dependence on institutional funds is unchanged from the Coluzzi report on services delivered in NCI-designated cancer centers [3]. Community-based treatment centers rely most heavily on institutional funds, and patient advocacy organizations rely most heavily on philanthropic funds.

Unfortunately, most organizations providing care to cancer patients have not adapted their educational materials and psychosocial/behavioral health services for a culturally or linguistically diverse patient population. Furthermore, respondents indicated that language and cultural barriers are noteworthy obstacles to providing psychosocial care for minority patients. With racial and ethnic minority populations nearing $50 \%$ of the US population, these findings are troubling and represent a major shortcoming in oncology care in the USA. They are also consistent with the IOM report suggesting that psychosocial care for minorities is inadequate [1].

Despite the recommendation that all cancer patients be screened for distress [5] and anticipation of the
American College of Surgeons' Commission on Cancer mandate for routine distress screening [6], only about half of the settings represented in this survey do so. Comprehensive cancer centers were relatively less likely to perform routine screening than communitybased centers. Furthermore, the method most endorsed for conducting screening-face-to-face interviewswhile being methodologically valid, is inefficient and labor-intensive. Indeed, the most commonly identified barrier to screening was insufficient qualified personnel. It could be that some survey respondents confused psychological assessment (more likely to be conducted by interview) with distress screening, and thus the prevalence of distress screening may be even lower than reported here. Lacking screening, many cancer patients' distress may go unrecognized, and thus, unaddressed.

Although it is encouraging that a wide variety of psychosocial services are provided in cancer settings and communities, the provision of these services is variable. Given that a majority of cancer patients remain unscreened for psychosocial distress, it is likely that patients are not being linked with appropriate resources to meet their needs. An area for growth is the implementation of routine screening for distress. This will likely be facilitated by the adoption of the Commission on Cancer accreditation standard in 2015 [6]. Another critical area for growth and improvement is the tailoring of psychosocial services and educational materials for a culturally diverse population.

\section{Acknowledgements}

The authors wish to express their gratitude to Laurel Couture, MSW, for her contributions to the preparation of this manuscript.

\section{References}

1. Institutes of Medicine. In Cancer Care for the Whole Patient: Meeting Psychosocial Health Needs, Adler NE, Page AEK (eds). The National Academies Press: Washington, DC, 2008.

2. Institutes of Medicine \& National Research Council. From Cancer Patient to Cancer Survivor: Lost in Transition. The National Academy Press: Washington, DC, 2006.

3. Coluzzi PH, Grant M, Doroshow JH et al. Survey of the provision of supportive care services at National Cancer Institute-Designated cancer centers. J Clin Oncol 1995; 13(3):756-764.

4. Holland JG. History of psycho-oncology: Overcoming attitudinal and conceptual barriers. Psychosom Med 2002;64: 206-221.

5. National Comprehensive Cancer Network. Clinical Practice Guidelines in Oncology, Distress Management, v. 1, 2011. www.nccn.org [Accessed 14 March 2011].

6. American College of Surgeons Commission on Cancer. Cancer Program Standards 2012, v. 1.0. http://www.facs. org/cancer/index [Accessed 1 December 2011]. 